\title{
Stem Cell Therapy for Cardiac Repair
}

\author{
John M. Collins ${ }^{1}$ and Brenda Russell ${ }^{1,2}$ \\ 1 Department of Bioengineering, University of Illinois at Chicago \\ 2Department of Physiology and Biophysics, University of Illinois at Chicago
}

\begin{abstract}
Heart regeneration via stem cell therapy could improve the functional outcome for millions of patients. A goal of cardiac stem cell research is to foster the engraftment of new, beating cardiac cells into the ischemic region of the heart after a myocardial infarction. The key elements of cell therapy for myocardial repair reviewed here are the source of cells and the mechanisms by which these cells improve cardiac function. Injection of stem cells into the heart of animals ignited the field by showing some functional cardiac improvement. Unfortunately, few injected cells are retained in the heart or become new beating myocardium, and clinical trials have shown moderate improvement of human heart function. The causes of the minimal functional improvement are still unknown but blood vessel formation (angiogenesis) or secretion of growth factors or cytokines are likely candidates. Cells appropriate for human therapy might be mesenchymal stem and progenitor cells from bone marrow or the heart itself. A more controversial cell source, embryonic stem cells, have a nearly unlimited self renewal potential and can differentiate into beating cardiac myocytes. However, all of these cell sources and the mechanisms of improvement need further research with differentiation of stem cells into functional cardiac cells a difficult but most beneficial hurdle to leap.
\end{abstract}

\section{Introduction}

There are currently five million heart failure patients in the United States ${ }^{1}$ for whom recovery of cardiac function could prove beneficial. A goal of cardiac stem cell research is to foster the engraftment of new, beating cardiac myocytes into the ischemic region of the heart after a myocardial infarction. Differentiation of cells into cardiac myocytes that are both mechanically and electrically coupled to the healthy cardiac myocytes is one way to achieve this goal. However, recent clinical trials indicate that other mechanisms probably predominate including stem cell differentiation into cells of vascular lineage resulting in better blood supply to the ischemic region. Another biologically useful action is the release of cytokines and growth factors from the injected cells leading to reduced cell death or enhanced recovery of host ventricular muscle cells. Growth factors may also help stem cells migrate into the damaged heart or activate a local population of stem cells residing within the heart.

The cell sources and mechanisms of cardiac improvement are two key elements of stem cell therapy reviewed here. Although the initial excitement surrounding cell-based therapy focused on stem cell differentiation into cardiac myocytes, clinical trials have shown that the vast majority of injected cells are not retained in the heart. ${ }^{2}$ Thus, injection of stem cells has not yet significantly improved heart function.

Correspondence to: Brenda Russell Ph.D., Department of Physiology and Biophysics (M/C 901), University of Illinois at Chicago, 835 S. Wolcott Avenue, Chicago, IL 60612-7342. E-mail: E-mail: russell@uic.edu, Telethon: 312 413-0407, Fax: 312-996-6312. 


\section{Sources of cells}

The optimal source of cells for regenerating damaged myocardium is a topic of intense research. Initial animal experiments using skeletal muscle precursor cells showed a general improvement of heart function. Specifically, skeletal myoblasts which were transplanted into the infarcted myocardium of rabbits remained viable and improved myocardial performance. ${ }^{3}$ However, later clinical trials showed these cells were not electrically coupled to the native heart cells and in some cases caused ventricular arrhythmias. Indeed, one would not expect electrical coupling of these cells since they lack the ability to make gap junctions. More recently, stem cells isolated from bone marrow, embryonic stem cells and resident cardiac stem cells have been used. High quality reviews on stem cell therapies for the heart can be consulted for detailed information. $4,5,6$

\section{Bone marrow cells - mesenchymal stem cells and endothelial progenitor cells}

The most prevalent source of cells used in clinical studies has been from bone marrow. Bone marrow cells as well as subpopulations from within the bone marrow including multipotent mesenchymal stem cells and endothelial progenitor cells have been studied. These subpopulations are sorted according to their expression of membrane receptor proteins, so called cell-surface markers. Whole bone marrow cell populations can be incubated with antibodies directed against cell-surface markers and attached to either magnetic beads or fluorescent tags. Magnetic columns or flow cytometry are then used to isolate the cells of interest from the whole population. In this fashion, mesenchymal stem and endothelial progenitor cells can be isolated from the bone marrow. However, a principal problem is that the cell-surface markers that determine a true cardiac stem cell have not yet been defined. As a result, there are a vast number of possible combinations of the various markers and cell subpopulations to be studied. It is not surprising that the outcomes of therapy have been variable since the subpopulations yielded from lab to lab have not been standardized. Currently, stem cells from bone marrow are usually identified first by the absence of surface markers of more committed hematopoietic lineages (termed "lineage negative"). Then they are further sorted by surface markers for mesenchymal stem cells (including but not limited to c-kit and sca-1) and endothelial progenitor cells (including but not limited to c-kit, vascular endothelial growth factor receptor-2, AC133, CXCR4 and CD34). ${ }^{7}$ Mesenchymal stem cells have also been separated from whole bone marrow by their ability to adhere to plastic substrates, justifying another name given to them - plastic adherent cells.

A popular model for testing the efficacy of stem cell therapy is to induce a myocardial infarct in a rodent and determine whether there is improved function after treatment. Preliminary studies indicated that lineage negative bone marrow cells injected into the border zone of an infarct regenerate the myocardium in mice. Regeneration of heart tissue was observed in $40 \%$ of the treated mice, and new cells were found in $68 \%$ of the infarcted portion of the ventricle. 8 This new heart tissue consisted of proliferating myofibroblasts and vascular structures as determined convincingly from tissue sections. The inability of other groups to obtain similar results has led to many questions about this early report. ${ }^{9}$ However, these initial studies have led to many of the clinical studies to date.

Both whole, unselected bone marrow cells and presorted mesenchymal stem cells from a patient's own bone marrow (i.e. autologous cells) have been used in human clinical trials. Mesenchymal stem cells from bone marrow retain their ability to differentiate into bone, cartilage, adipose tissue and skeletal muscle. ${ }^{10,11}$ Autologous cells have improved cardiac performance in both animals and humans with recent randomized human studies supporting the safety and feasibility of autologous bone marrow cell therapy. Two studies showed moderate but statistically significant improvement of left ventricular function at 3 and 4 months after intracoronary administration of these cells. ${ }^{12,13}$ These findings were not confirmed in 
a third study. ${ }^{14}$ The reasons for the different results are not known. It is clear that there are problems with intracoronary injection of cells because very few of the cells injected into the heart become cardiac myocytes. A major emphasis of current research is to define the subpopulation of those very rare cells in the bone marrow that may have the capacity to make contractile heart cells. To date, however, this has not been successful.

Endothelial progenitor cells are a second subpopulation within the bone marrow that can promote vasculogenesis leading to vessel regrowth, thereby improving oxygenation to tissue after damage. ${ }^{7}$ Preclinical trials indicate that endothelial progenitor cells contribute to $1-25 \%$ of vessel formation after ischemic injury for several diseases. 7 Additionally, endothelial progenitors may secrete growth factors and paracrine signals that prevent cardiac myocyte cell death. ${ }^{15}$ These cells are capable of vascular differentiation and secretion of growth factors promoting angiogenesis and increasing blood supply to the damaged heart. However, differentiation of stem cells into functional cardiac myocytes is a main goal of stem cell therapy, and the following cell types may be most promising to accomplish this.

\section{Embryonic Stem Cells}

Although ethical and political considerations have blunted research on human embryonic stem cells, they are an attractive source for cell-based therapy and have been used worldwide. Characterized as pluripotent, embryonic stem cells have a nearly unlimited self renewal and can differentiate into beating cardiac myocytes as demonstrated by electrical and mechanical coupling to the host myocardium (see the first paper in this series). However, one of their biggest limitations is that cancerous teratomas may form due to the ability of embryonic stem cells to divide and differentiate into almost any cell type. Thus, clinical trials with this cell source are lagging behind those for other kinds of stem cells. However, differentiation of embryonic stem cells prior to implantation may both prevent oncogenesis and yield a pure cardiac myocyte population. Field and colleagues ${ }^{16}$ have used genetic selection to isolate cells that were $99.6 \%$ positive for heart muscle myosin. Embryonic stem cells were transfected with a fusion gene including the cardiac specific alpha-cardiac myosin heavy chain promoter, differentiated in vitro, and then selected. Implantation of these cells resulted in stable grafts in animals. Although there have yet to be clinical trials using embryonic stem cells, electromechanical coupling has been demonstrated between enriched cardiac cells derived from human embryonic stem cells that were transplanted into rat hearts, leading to increased myocardial wall thickness. 17

\section{Resident cardiac stem cells}

Contrary to the longstanding thought that the heart is a terminally differentiated organ incapable of self regeneration or repair, recent data suggest that the heart contains stem cells. ${ }^{18}$ The idea that heart growth can only be achieved through hypertrophy was challenged with data indicating that niches within the heart contain cells that divide and can replace damaged cardiac myocytes. These resident cardiac stem cells express the cell-surface marker, c-kit, and possess fundamental properties of stem cells in that they are self-renewing and capable of differentiation into multiple cell types. ${ }^{19}$ This subset of cells has been shown to migrate to damaged regions of the heart and generate new cardiac myocytes in rats, hence improving cardiac function. 20

\section{Mechanism of functional cardiac improvement}

\section{Differentiation of stem cells}

Currently there is no consensus among investigators with regard to the capability of bone marrow cells to differentiate into functional cardiac myocytes. The results of some studies suggest that stem cells from bone marrow can differentiate into cardiac myocytes, ${ }^{8}$ while the 
results of others do not. ${ }^{9}$ Although there is no evidence from human clinical trials that bone marrow cells injected into the heart differentiate into cardiac myocytes there is evidence in animals that this occurs occasionally.

In vitro co-culture studies of stem cells with primary cells and in vivo injection of stem cells in animals have illustrated the ability of stem cells to differentiate into cardiac cells. Primary cardiac myocytes harvested from neonatal rats induce cardiac myogenic differentiation of endothelial progenitor cells 21,22 and mesenchymal stem cells. ${ }^{23,24}$ Additionally, mesenchymal stem cells injected into pig 25 or sheep ${ }^{26}$ hearts days after myocardial infarction, express muscle-specific proteins, result in long-term engraftment, reduce scar formation and improve cardiac function. ${ }^{25}$ The engrafted mesenchymal stem cells express proteins found in cardiac myocytes, as well as vascular endothelium and smooth muscle. ${ }^{26}$ Although expression of cardiac proteins in these cells is a good first indication of cardiac differentiation, there is little evidence that these cells differentiate into beating heart cells in vivo. It is likely that injection of stem cells into the mechanically active heart may not be the best approach for cardiac regeneration.

The activation of a population of stem cells that reside within the heart may be a more promising possibility for heart regeneration. Results of some studies demonstrate "proof of principle" that a population of resident cardiac stem cells found within the heart can differentiate toward the cardiac lineage. Current research is directed towards understanding how this occurs and how to increase the number of these highly desirable functional cells. Importantly, the number and percentage of dividing cardiac stem cells were shown to be greater in acutely infarcted hearts and hearts with end-stage cardiomyopathy as compared to normal cardiac tissue. ${ }^{27}$

Additionally, cardiac stem cells have an increased commitment to the cardiac myocyte, smooth muscle and endothelial cell lineages in the infarcted and end-stage hearts versus normal hearts. 27 Resident cardiac stem cells could be the best cell source for heart muscle regeneration if the proportion that becomes beating heart cells could be enhanced.

None of the clinical studies mentioned above ${ }^{11-13}$ provide evidence that intracardiac injection of cells isolated from bone marrow differentiate into cardiac muscle cells. However, differentiation of stem cells into vascular cells is commonly observed and represents another mechanism by which heart function can be improved after myocardial infarction. It is likely that clinical studies showing functional improvements associated with the direct delivery of bone marrow cells to the heart are mainly due to the secretion of factors that increase angiogenesis. ${ }^{28,29} \mathrm{~A}$ recent paper suggests that cardiac stem cells engrafted within host rat myocardium, divided and differentiated into endothelial cells, smooth muscle cells, and few cardiac myocytes. ${ }^{30}$ Most of the cells formed coronary arteries, arterioles and capillaries leading to increased myocardial blood flow, thus improving function. ${ }^{30}$ In this particular study, prior to injection, the cardiac stem cells were activated with insulin-like growth factor 1 and hepatocyte growth factor. These and several other factors are known to influence stem cell function in the heart.

\section{Secreted factors from stem cells}

Once scientists can develop the right kind of pre-myocyte stem cells, they will still need to direct them to where they are needed for repair. This process of direction is called "homing" or the movement of cells towards the injured region where they adhere, engraft and are not flushed away. The homing of stem cells from tissues such as bone marrow to the heart in response to various factors has been explored. Earlier animal studies showed that direct injection of two signaling proteins: stem cell factor and granulocyte-colony stimulating factor, facilitated the homing of bone marrow cells to the infarct border zone. ${ }^{31}$ Mortality was reduced with improvement seen in infarct size, ejection fraction and hemodynamics. In another study, mice treated with granulocyte-colony stimulating factor had a higher number and better 
migration of resident cardiac stem cells into the infarct zone. ${ }^{32}$ Finally, vascular endothelial growth factor increased angiogenesis and homing of endothelial progenitors from the bone marrow. ${ }^{33}$ Thus, patients with end-stage coronary artery disease were able to walk longer before reporting angina, a modest trend towards improved left ventricular function. These results suggest that it is very likely that the regenerative capability of bone marrow and cardiac stem cells requires a combination of growth factors.

\section{Conclusion}

Of the many challenges for successful cardiac regeneration with stem cell therapy, the cell source is the most important. The primitive stem characteristics and differentiation potential of embryonic stem cells make them the ideal candidate for treatment of heart disease. However, their clinical use is currently limited due to the potential to form cancer cells and the debate about ethical considerations. At this time, bone marrow cells have been most used in clinical trials and have shown potential to improve heart function. However, the exact mechanism(s) responsible for improved function is unclear. Reasons for different outcomes among studies may include the use of various subpopulations of mesenchymal stem and endothelial progenitor cells. For example, one subpopulation might release growth factors beneficial to the undamaged cells of the heart while another, albeit in low frequency, might differentiate towards the cardiac myocyte lineage. It is clear that soluble cues from the microenvironment of the heart play a key role in the differentiation and control of stem cell function. Physical cues imposed by the mechanically dynamic heart likely play a large role and tissue engineering approaches (see the third paper in this series) may hold the key to better differentiation of stem cells into cardiac myocytes, homing of stem cells to the damaged cardiac tissue and controlled division of the cells.

To date, the formation of vasculature has proven a beneficial outcome of stem cell therapy. However, a main goal of cardiac differentiation of stem cells in vivo has not yet been realized. The body readily builds new vessels and uses its own adult progenitor stem cells to accomplish this; however researchers have been unsuccessful in engrafting injected stem cells in the heart and in producing enough new contractile myocytes to improve heart function significantly. There is a high therapeutic potential for resident cardiac stem cells, and increasing the numbers of these cells may be the most practical way to enhance cardiac regeneration in people with heart disease.

Stem cell therapy is a very exciting field with great potential for heart regeneration. Early clinical trials have shown moderate improvement in heart function after treatment and research focused on potentiating these improvements is important for future success of stem cell therapy. Although the field has progressed rapidly to clinical trials using various populations of stem cells, much more research is needed to characterize the potential of the various cell populations used for stem cell therapy and to understand the basic science regulating the differentiation, proliferation and integration with the host heart.

\section{Acknowledgements}

The work was supported by NIH (HL 62426); State of Illinois funds for Regenerative Medicine; AHA predoctoral fellowship (0815535G).

\section{References}

1. American Heart Association. Heart Disease and Stroke Statistics-2006 Update. Dallas, Tex: American Heart Association; 2006.

2. Hofmann M, Wollert KC, Meyer GP, et al. Monitoring of Bone Marrow Cell Homing Into the Infarcted Human Myocardium. Circ 2005;111(17):2198-2202. 
3. Taylor DA, Atkins BZ, Hungspreugs P, et al. Regenerating functional myocardium: improved performance after skeletal myoblast transplantation. Nat Med 1998;4(8):929-933. [PubMed: 9701245]

4. Seegers VFM, Lee RT. Stem-cell therapy for cardiac disease. Nature 2008;451(21):938-942.

5. Murry CE, Field LJ, Menasché P. Cell-based cardiac repair: reflections at the 10-year point. Circulation 2005;112(20):3174-3183. [PubMed: 16286608]

6. Laflamme MA, Murry CE. Regenerating the heart. Nat Biotechnol 2005;23(7):845-856. [PubMed: 16003373]

7. Young PP, Vaughan DE, Hatzopoulos AK. Biologic properties of endothelial progenitor cells and their potential for cell therapy. Prog Cardiovasc Dis 2007;49(6):421-429. [PubMed: 17498522]

8. Orlic D, Kajstura J, Chimenti S, et al. Bone marrow cells regenerate infarcted myocardium. Nature 2001;410(6829):701-705. [PubMed: 11287958]

9. Murry CE, Soonpaa MH, Reinecke H, et al. Haematopoietic stem cells do not transdifferentiate into cardiac myocytes in myocardial infarcts. Nature 2004 Apr 8;428(6983):664-668. [PubMed: 15034593]

10. Pittenger MF, Mackay AM, Beck SC, et al. Multilineage potential of adult human mesenchymal stem cells. Science 1999;284(5411):143-147. [PubMed: 10102814]

11. Ferrari G, Cusella-De Angelis G, Coletta M, et al. Muscle regeneration by bone marrow-derived myogenic progenitors. Science 1998;279(5356):1528-1530. [PubMed: 9488650]

12. Assmus B, Honold J, Schachinger V, et al. Transcoronary transplantation of progenitor cells after myocardial infarction. N Engl J Med 2006;355(12):1222-1232. [PubMed: 16990385]

13. Schachinger V, Erbs S, Elsasser A, et al. Intracoronary bone marrow-derived progenitor cells in acute myocardial infarction. N Engl J Med 2006;355(12):1210-1221. [PubMed: 16990384]

14. Lunde K, Solheim S, Aakhus S, et al. Intracoronary injection of mononuclear bone marrow cells in acute myocardial infarction. N Engl J Med 2006;355(12):1199-1209. [PubMed: 16990383]

15. Narmoneva DA, Vukmirovic R, Davis ME, Kamm RD, Lee RT. Endothelial cells promote cardiac myocyte survival and spatial reorganization: implications for cardiac regeneration. Circ 2004;110 (8):962-968.

16. Klug MG, Soonpaa MH, Koh GY, Field LJ. Genetically selected cardiomyocytes from differentiating embryonic stem cells form stable intracardiac grafts. J Clin Invest 1996;98(1):216-224. [PubMed: 8690796]

17. Laflamme MA, Gold J, Xu C, et al. Formation of human myocardium in the rat heart from human embryonic stem cells. Am J Pathol 2005;167(3):663-671. [PubMed: 16127147]

18. Urbanek K, Cesselli D, Rota M, et al. Stem cell niches in the adult mouse heart. Proc Natl Acad Sci U S A 2006;103(24):9226-9231. [PubMed: 16754876]

19. Beltrami AP, Barlucchi L, Torella D, et al. Adult cardiac stem cells are multipotent and support myocardial regeneration. Cell 2003;114(6):763-776. [PubMed: 14505575]

20. Gonzalez A, Rota M, Nurzynska D, et al. Activation of cardiac progenitor cells reverses the failing heart senescent phenotype and prolongs lifespan. Circ Res 2008;102(5):597-606. [PubMed: 18202313]

21. Condorelli G, Borello U, De Angelis L, et al. Cardiomyocytes induce endothelial cells to transdifferentiate into cardiac muscle: implications for myocardium regeneration. Proc Natl Acad Sci U S A 2001;98(19):10733-10738. [PubMed: 11535818]

22. Badorff C, Brandes RP, Popp R, et al. Transdifferentiation of blood derived human adult endothelial progenitor cells into functionally active cardiomyocytes. Circ 2003;107(7):1024-1032.

23. Shim WS, Jiang S, Wong P, et al. Ex vivo differentiation of human adult bone marrow stem cells into cardiomyocyte-like cells. Biochem Biophys Res Commun 2004;324(2):481-488. [PubMed: 15474453]

24. Li X, Yu X, Lin Q, et al. Bone marrow mesenchymal stem cells differentiate into functional cardiac phenotypes by cardiac microenvironment. J Mol Cell Cardiol 2007;42(2):295-303. [PubMed: 16919679] 
25. Amado LC, Saliaris AP, Schuleri KH, et al. Cardiac repair with intramyocardial injection of allogeneic mesenchymal stem cells after myocardial infarction. Proc. Natl Acad. Sci. USA 2005;102(32): 11474-11479. [PubMed: 16061805]

26. Liechty KW, MacKenzie TC, Shaaban AF, et al. Human mesenchymal stem cells engraft and demonstrate site-specific differentiation after in utero transplantation in sheep. Nat Med 2000;6(11): 1282-1286. [PubMed: 11062543]

27. Urbanek K, Torella D, Sheikh F, et al. Myocardial regeneration by activation of multipotent cardiac stem cells in ischemic heart failure. Proc Natl Acad Sci U S A 2005;102(24):8692-8697. [PubMed: 15932947]

28. Fazel S, Cimini M, Chen L, et al. Cardioprotective c-kit+ cells are from the bone marrow and regulate the myocardial balance of angiogenic cytokines. J Clin Invest 2006;116(7):1865-1877. [PubMed: 16823487]

29. Kawamoto A, Iwasaki H, Kusano K, et al. CD34-positive cells exhibit increased potency and safety for therapeutic neovascularization after myocardial infarction compared with total mononuclear cells. Circ 2006;114(20):2163-2169.

30. Tillmanns J, Rota M, Hosoda T, et al. Formation of large coronary arteries by cardiac progenitor cells. Proc Natl Acad Sci U S A 2008;105(5):1668-1673. [PubMed: 18216245]

31. Orlic D, Kajstura J, Chimenti S, et al. Mobilized bone marrow cells repair the infarcted heart, improving function and survival. Proc Natl Acad Sci U S A 2001;98(18):10344-10349. [PubMed: 11504914]

32. Brunner S, Huber BC, Fischer R, et al. G-CSF treatment after myocardial infarction: Impact on bone marrow-derived vs cardiac progenitor cells. Exp Hematol. 2008[Epub ahead of print].

33. Yoon YS, Johnson IA, Park JS, Diaz L, Losordo DW. Therapeutic myocardial angiogenesis with vascular endothelial growth factors. Mol Cell Biochem 2004;264(1-2):63-74. [PubMed: 15544036] 